\title{
Incremental stress and microstructural response of granular soils under undrained axisymmetric deformation
}

\author{
N. Kumar, S. Luding \& V. Magnanimo \\ MultiScale Mechanics, CTW, MESA+ \\ University of Twente, Enschede, Netherlands
}

\begin{abstract}
The influence of the micromechanics on the response of stress and fabric on strain is an essential part of a constitutive model for granular matter, because it contains the information of how different deformation paths (history) affect the actual mechanical state of the system. Here, we study a granular aggregate that is first isotropically compressed and then subjected to large axisymmetric strain extension at constant volume. At various axial strain amplitudes, we determine the stress and fabric incremental response envelopes that result from the application of strain-probes with identical total magnitude but different contribution of isotropic relative to deviatoric strain (which span an orthogonal two-dimensional subset in "strain-space", in which the "direction-angle" defines the relative magnitude of the two contributions). For very small strain increments the fabric response is null in shear direction, i.e. the structure does not change and the elastic stress-response is probed. Larger strain are associated with rearrangements of contacts probing the combined plastic stress- and fabric-response. With increasing shear-induced anisotropy, the centers, inclinations and the thickness of the response envelopes in the stress-space change, involving the maximum and minimum stiffness.
\end{abstract}

\section{INTRODUCTION}

Granular materials behave differently from usual solids or fluids and show peculiar mechanical properties like dilatancy, history dependence, ratcheting and anisotropy, see (de Gennes 1999) and references therein. The behavior of these materials is highly non-linear and involves plasticity also for very small strain due to rearrangements of the elementary particles (Goddard 1990, Sibille 2009, Bardet 1994, Kumar 2014). The concept of an initial purely elastic regime (small strain) for granular assemblies is an issue still under debate in the mechanical and geotechnical communities (Einav 2012). Recent works (Ezaoui 2009, Zhao 2013, La Ragione 2012) show that along with the macroscopic properties (stress and volume fraction) also the structure, quantified by the fabric tensor (Luding 2005) plays a crucial role, as it characterizes, on average, the geometric arrangement of contacts. In particular, when the material is sheared, anisotropy in the contact network develops, as related to the opening and closing of contacts, restructuring, and the creation and destruction of force-chains. The anisotropic state is at the origin of interesting observations on wave propagation in sheared granular media.

In this work we use the Discrete Element Method
(DEM) to study frictionless, anisotropic granular assemblies and focus on their elastic and elasto-plastic behavior. In order to investigate the evolution of the incremental response as a function of the loading direction, we perform so-called strain probing tests along an axisymmetric deformation path, resembling what happens in physical experiments (Muir Wood 2006). The directional-dependent data are collected in stress and fabric response envelopes as defined in (Gudheus 1979) in the case of both small (elastic) and big (elasto-plastic) probes. In the case of a finite assembly of particles, in simulations, a finite elastic regime can always be detected and the elastic stiffnesses can be measured by means of an actual, very small, strain perturbation (Magnanimo 2008). By isolating elasticity we aim to identify the kinematics at the microscale that lead to either macroscopic elasticity or plasticity. The final goal is to improve the understanding of anisotropic elasticity in particle systems and to guide further developments for new constitutive models based on microstructure informations.

\section{NUMERICAL SIMULATION}

The Discrete Element Method (DEM) (Luding 2005) helps to better understand in detail the deformation be- 
havior of particle systems. At the basis of DEM are force laws that relate the interaction force to the overlap of two particles. Neglecting tangential forces, if all normal forces $\mathbf{f}_{i}$ acting on particle $i$, from all sources, are known, the problem is reduced to the integration of Newton's equations of motion for the translational degrees of freedom: $\frac{\mathrm{d}}{\mathrm{d} t}\left(m_{i} \mathbf{v}_{i}\right)=\mathbf{f}_{i}+m_{i} \mathbf{g}$, with the mass $m_{i}$ of particle $i$, its position $\mathbf{r}_{i}$, velocity $\mathbf{v}_{i}\left(=\dot{\mathbf{r}}_{i}\right)$ and the resultant force $\mathbf{f}_{i}=\sum_{c} \mathbf{f}_{i}{ }^{c}$ acting on it due to contacts with other particles or with the walls, and the acceleration due to gravity, $\mathrm{g}$ (which is neglected in this study).

\subsection{Contact model}

For the sake of simplicity, the linear visco-elastic contact model for the normal component of force is used. The simplest normal contact force model is given by $f^{n}=k \delta+\gamma \dot{\delta}$, where $k$ is the spring stiffness, $\gamma$ is the contact viscosity parameter, $\delta=$ $\left(d_{i}+d_{j}\right) / 2-\left(\mathbf{r}_{i}-\mathbf{r}_{j}\right) . \hat{\mathbf{n}}$ is the overlap between two interacting species $i$ and $j$ with diameters $d_{i}$ and $d_{j}$, $\hat{\mathbf{n}}=\left(\mathbf{r}_{i}-\mathbf{r}_{j}\right) /\left|\left(\mathbf{r}_{i}-\mathbf{r}_{j}\right)\right|$ and $\dot{\delta}$ is the relative velocity in the normal direction. In order to reduce dynamical effects and shorten relaxation times, an artificial viscous background dissipation force $\mathbf{f}_{b}=-\gamma_{b} \mathbf{v}_{i}$ proportional to the moving velocity $\mathbf{v}_{i}$ of particle $i$ is added, resembling the damping due to a background medium, as e.g. a fluid.

The standard simulation parameters are, $N=$ $9261\left(=21^{3}\right)$ particles with average radius $\langle r\rangle=1$ $[\mathrm{mm}]$, density $\rho=2000\left[\mathrm{~kg} / \mathrm{m}^{3}\right]$, elastic stiffness $k=$ $10^{8}\left[\mathrm{~kg} / \mathrm{s}^{2}\right]$, particle damping coefficient $\gamma=1[\mathrm{~kg} / \mathrm{s}]$, background dissipation $\gamma_{b}=0.1[\mathrm{~kg} / \mathrm{s}]$. Note that the polydispersity of the system is quantified by the width $\left(w=r_{\max } / r_{\min }=3\right)$ of a uniform size distribution, where $r_{\max }$ and $r_{\min }$ are the radii of the biggest and smallest particles respectively. For details about other time scales present in the system, see (Imole 2013, Kumar 2014).

\subsection{Macroscopic (tensorial) quantities}

Here, we define averaged tensorial macroscopic quantities - including strain-, stress- and fabric (structure) tensors - that provide information about the state of the packing and reveal interesting bulk features.

By speaking about the strain-rate tensor $\dot{\mathbf{E}}$, we refer to the external strain that we apply to the sample. The isotropic part of the infinitesimal strain tensor $\varepsilon_{\mathrm{v}}$ is defined as: $\varepsilon_{\mathrm{v}}=\dot{\varepsilon}_{\mathrm{v}} \mathrm{dt}=-\left(\varepsilon_{x x}+\varepsilon_{y y}+\varepsilon_{z z}\right) / 3=$ $\operatorname{tr}(-\mathbf{E}) / 3=\operatorname{tr}(-\dot{\mathbf{E}} \mathrm{d} t) / 3$, where $\varepsilon_{\alpha \alpha}=\dot{\varepsilon}_{\alpha \alpha} \mathrm{dt}$ with $\alpha \alpha=$ $x x$, yy and $z z$ as the diagonal components of the tensor in the Cartesian $x-y-z$ reference system. The trace integral of $3 \varepsilon_{\mathrm{v}}$ is denoted as $\varepsilon_{v}$, the true or logarithmic volumetric strain, i.e., the volume change of the system, relative to the initial reference volume, $V_{0}$.
On the other hand, from DEM simulations, one can measure the 'static' stress in the system as

$\boldsymbol{\sigma}=(1 / V) \sum_{c \in V} \mathbf{l}^{c} \otimes \mathbf{f}^{c}$,

averaged over all the contacts in the volume $V$ of the dyadic products between the contact force $\mathbf{f}^{c}$ and the branch vector $\mathbf{l}^{c}$, where the contribution of the kinetic fluctuation energy has been neglected (Luding 2005, Imole 2013). The isotropic component of the stress is the pressure $P=\operatorname{tr}(\boldsymbol{\sigma}) / 3$.

In order to characterize the geometry/structure of the static aggregate at microscopic level, we measure the fabric tensor, defined as

$\mathbf{F}=\frac{1}{V} \sum_{\mathcal{P} \in V} V^{\mathcal{P}} \sum_{c \in \mathcal{P}} \mathbf{n}^{c} \otimes \mathbf{n}^{c}$,

where $V^{\mathcal{P}}$ is the particle volume for particle $\mathcal{P}$, which lies inside the averaging volume $V$, and $\mathbf{n}^{c}$ is the normal unit branch-vector pointing from center of particle $\mathcal{P}$ to contact $c$ (Luding 2005, Kumar 2014).

We choose here to describe each symmetric second order tensor $\mathrm{Q}$, in terms of its isotropic part (first invariant) and the second $J_{2}$ and third $J_{3}$ invariants of the deviator: $J_{2}=\frac{1}{2}\left[\left(Q_{1}^{D}\right)^{2}+\left(Q_{2}^{D}\right)^{2}+\left(Q_{3}^{D}\right)^{2}\right]$ and $J_{3}=$ $\operatorname{det}\left(\mathbf{Q}^{D}\right)=Q_{1}^{D} Q_{2}^{D} Q_{3}^{D}$, with $Q_{1}^{D}, Q_{2}^{D}$ and $Q_{3}^{D}$ eigenvalues of the deviatoric tensor $\mathbf{Q}^{D}=\mathbf{Q}-(1 / 3) \operatorname{tr}(\mathbf{Q}) \mathbf{I}$. The deviatoric part is described with a single scalar quantity by using the second invariant of the deviatoric tensor, $Q_{\mathrm{dev}}= \pm \sqrt{2 J_{2}}$, where the sign relates to either axial compression $(+)$ or extension $(-)$, and the deviators $\varepsilon_{\mathrm{dev}}, \sigma_{\mathrm{dev}}$ and $F_{\mathrm{dev}}$ refer to strain $-\mathbf{E}$, stress $\sigma$ and fabric $\mathbf{F}$ respectively (Kumar 2014). Note that in this work we use $k^{*}=k /(2\langle r\rangle)$ to non-dimensionalize the stress, i.e. $\boldsymbol{\sigma}^{*}=\boldsymbol{\sigma} / k^{*}$.

\section{VOLUME CONSERVING (UNDRAINED) AXISYMMETRIC TEST}

In this section, we first describe the preparation procedure and then the details of the numerical shear test.

The initial configuration is such that spherical particles are randomly generated, with low volume fraction and rather large random velocities in a periodic 3D box, such that they have sufficient space and time to exchange places and to randomize themselves. This granular gas is then compressed isotropically, to a target volume fraction $\nu_{0}=0.640$, sightly below the isotropic jamming volume fraction $\nu_{c} \approx 0.658$ and the system is let to relax toward equilibrium (Imole 2013, Kumar 2014). The relaxed state is further compressed (loading) isotropically from $\nu_{0}$ to a higher volume fraction of $\nu_{\max }=0.82$ (Imole 2013, Kumar 2014). We take this isotropic sample at $\nu_{\max }$ and shear the sample keeping the total volume constant, by applying the 


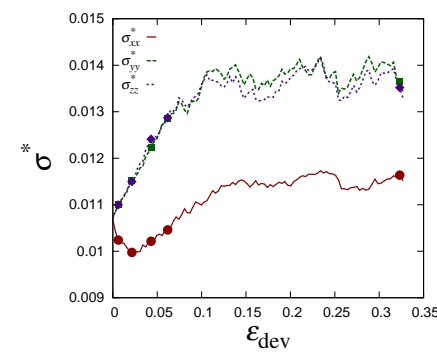

(a)

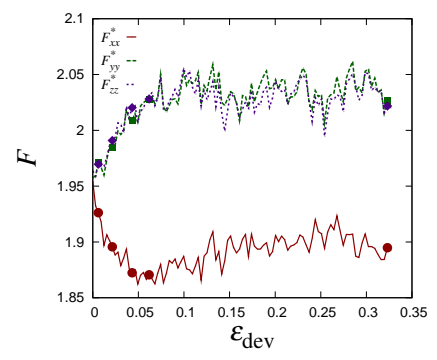

(b)
Figure 1: Evolution of (a) stress and (b) fabric components with applied shear strain $-\varepsilon_{\mathrm{dev}}$ in the case of undrained axisymmetric deformation path at volume fraction $\nu=0.82$. The corresponding points represent the states chosen along the shear path at $-\varepsilon_{\mathrm{dev}}=0.006,0.021,0.043,0.068$ and 0.32 , where small strain perturbations probes are applied.

strain-rate tensor

$\dot{\mathbf{E}}=\dot{\varepsilon}_{\operatorname{dev}}\left[\begin{array}{ccc}1 & 0 & 0 \\ 0 & -1 / 2 & 0 \\ 0 & 0 & -1 / 2\end{array}\right]$,

where $x-$ is the axial extension direction and $\dot{\varepsilon}_{\mathrm{dev}}=$ $28.39\left[\mu \mathrm{s}^{-1}\right]$ the strain-rate (compression $<0$ ) amplitude applied to the walls. Our volume conserving shear test, resembles the undrained triaxial test typical in geotechnical practice (Zhao 2013).

The evolution of the components of (nondimensional) stress $\boldsymbol{\sigma}^{*}$ during shear, is shown in Fig. 1(a), as function of the deviatoric strain $-\varepsilon_{\mathrm{dev}}$. The tensile stress $\sigma_{x x}^{*}$ first decreases and then slightly increases with applied strain, while the compressive components $\sigma_{y y}^{*}$ and $\sigma_{z z}^{*}$, increase (due to compression) until an asymptote is reached. A similar behavior is observed for fabric in Fig. 1(b), with $F_{x x}$ decreasing and $F_{y y}, F_{z z}$ increasing with strain.

\section{RESPONSE ENVELOPE}

We now want to study the evolution of the incremental behavior during shear as function of the loading direction. We choose five different initial states along the deviatoric path in Fig. 1, the first one being near isotropic and last one highly anisotropic, and apply sufficient relaxation, so that the granular assemblies dissipate the kinetic energy they had during the original shearing path, even though it was very small. Further on we probe the samples, by applying small strain perturbations and measuring the incremental (stress and fabric) responses (Magnanimo 2008, Kumar 2014). For each state we apply several perturbations that have identical magnitude $\|\delta \varepsilon\|=$ $\sqrt{\left(\delta \varepsilon_{x x}\right)^{2}+\left(\delta \varepsilon_{y y}\right)^{2}+\left(\delta \varepsilon_{z z}\right)^{2}}$ and point in different directions of the strain space

$\delta \dot{\mathbf{E}} \mathrm{d} t=\|\delta \varepsilon\|\left[\begin{array}{ccc}\sin \theta & 0 & 0 \\ 0 & \frac{1}{\sqrt{2}} \cos \theta & 0 \\ 0 & 0 & \frac{1}{\sqrt{2}} \cos \theta\end{array}\right]$, where $\theta \in\left[0^{\circ}, 360^{\circ}\right]$ is the between the strain increment vector and the horizontal axis. Because the main path is deviatoric axisymmetric, Eq. (1), we apply incremental strains that resemble that type of symmetry for each $\theta$.

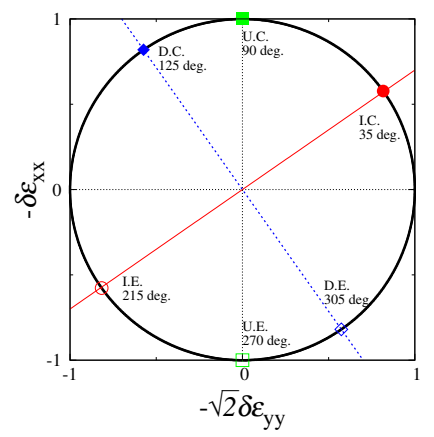

Figure 2: (a) Location of points of isotropic extension and compression (I.C. \& I.E. $35^{\circ} \& 125^{\circ}$ respectively - red line), uniaxial extension and compression (U.C. \& U.E. $90^{\circ} \& 270^{\circ}$ respectively) and deviatoric extension and compression (D.C. \& D.E. $125^{\circ} \& 305^{\circ}$ respectively - blue line) on the Rendulic plane of strain.

The response of the granular assembly is depicted by using the so-called incremental stress response envelope, defined as the image in the stress rate space of the unit sphere in the strain rate space (Gudheus 1979, Bardet 1994, Muir Wood 2006, Calvetti 2003, AlonsoMarroquín 2005). In the specific case of axisymmetric loading, the number of independent components of strain (and stress) reduces to two: the strain probes define a circle in the Rendulic plane of strain increments $\delta \varepsilon_{x x}: \sqrt{2} \delta \varepsilon_{y y} \equiv \sin \theta: \cos \theta$, and the response envelope can be visualized in the incremental stress plane $\delta \sigma_{x x}^{*}: \sqrt{2} \delta \sigma_{y y}^{*}$ (Wu 2000, Calvetti 2003). It is easy to show that an incremental linear behavior leads to an ellipse centered at the origin of the stress increment plane (Gudheus 1979). The distance of a point on the response envelope to the center of the ellipse characterizes the stiffness corresponding to a given direction of strain (directional stiffness) (Wu 2000), that is the envelope of stress provides a visual indication of the stiffness characteristics of the material. Any deviation from this kind of response, such as shift of the center of the envelope from the origin, strongly suggests some form of incremental non-linearity.

In figure 2 we give an example of the procedure and plot the strain circle, the stress response envelopes for the nearly isotropic configuration at $-\varepsilon_{\mathrm{dev}}=0.006$ and in the steady state configuration at $-\varepsilon_{\mathrm{dev}}=0.32$, all of them in the respective Rendulic planes. In Fig. 2 the special directions of purely isotropic, uniaxial, purely deviatoric volume conserving perturbations are highlighted. The same points are then located in the corresponding stress envelopes in Figs. 3(a)-3(e). Special features of the two envelopes can be detected. When a purely volumetric perturbation is applied, the stress response in Fig. 3(a) is, as expected, purely isotropic and corresponds to the major axis of the ellipse, such axis 


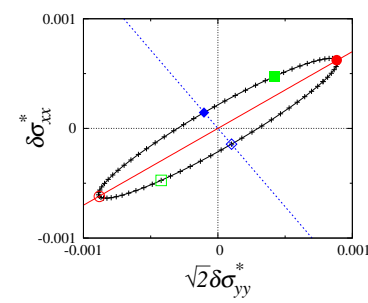

(a)

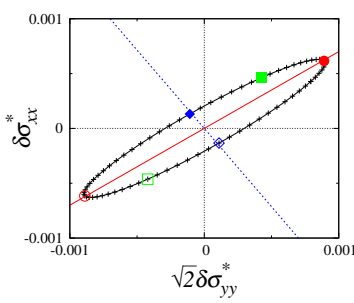

(b)

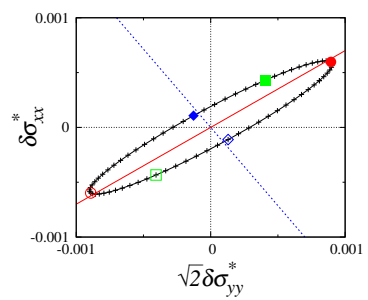

(c)

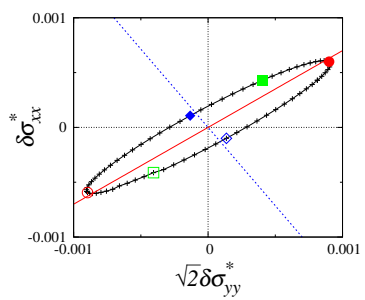

(d)

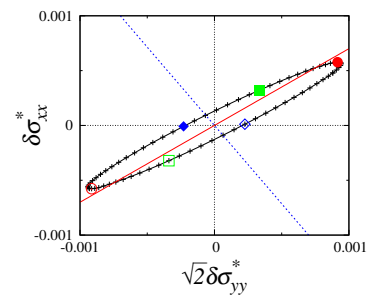

(e)

Figure 3: Evolution of the elastic stress response envelope with deviatoric strain applied to the sample, starting from nearly isotropic configuration to highly anisotropic state. Configurations (a)-(e) correspond to deviatoric strain $-\varepsilon_{\text {dev }}=0.006,0.021,0.043,0.068,0.32$ respectively. The applied strain increment is small, $\delta \varepsilon<10^{-4}$ and the response elastic. Points corresponds to the special directions, as shown in Fig. 2.

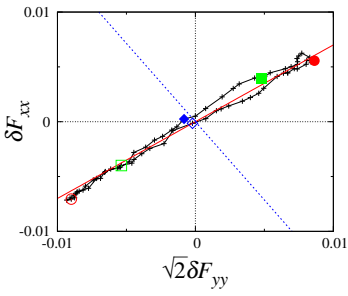

(a)

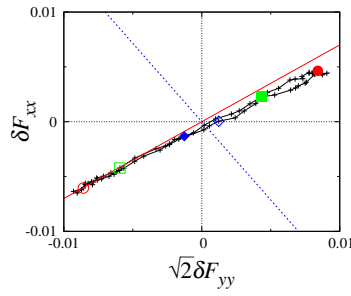

(b)

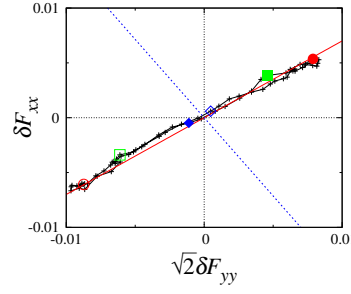

(c)

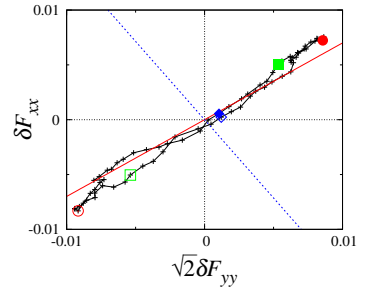

(d)

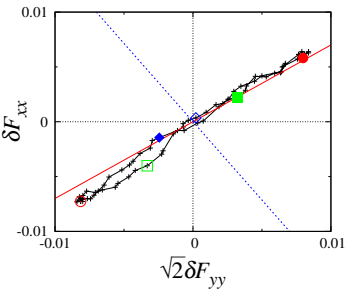

(e)

Figure 4: Evolution of the elastic fabric response envelope from the same configurations as in Fig. 3.

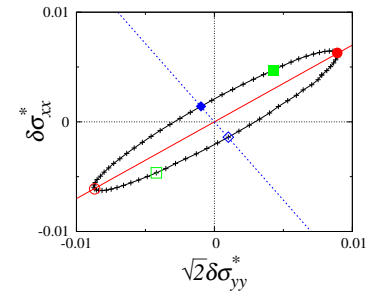

(a)

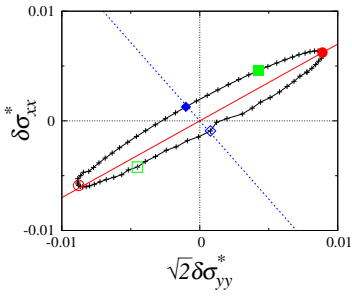

(b)

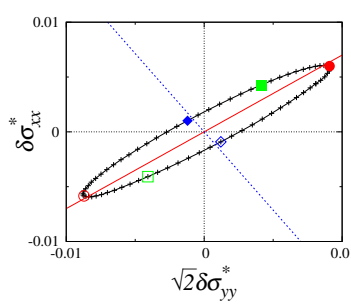

(c)

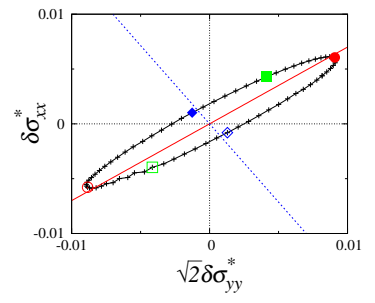

(d)

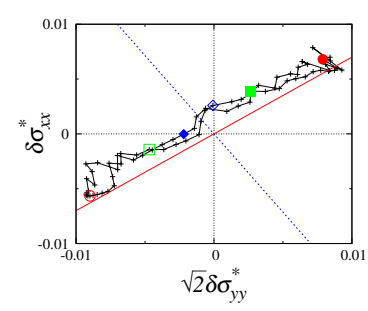

(e)

Figure 5: Evolution of the elasto-plastic stress response envelope from the same configurations as in Fig. 3, with higher strain increment $\delta \varepsilon>10^{-3}$ and response plastic.

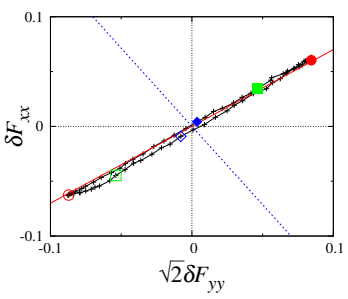

(a)

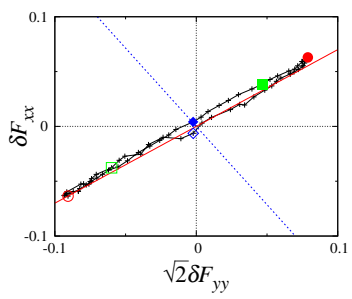

(b)

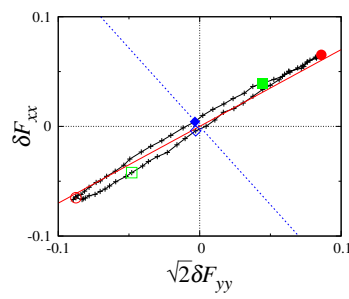

(c)

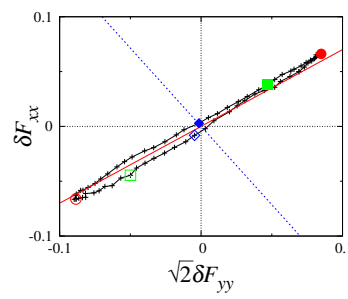

(d)

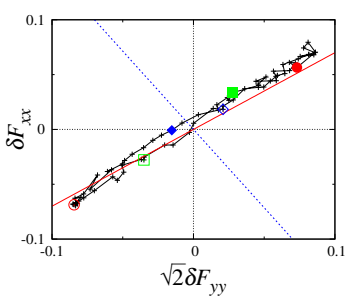

(e)

Figure 6: Evolution of the elasto-plastic fabric response envelope from the same configurations as in Fig. 5.

being a measure of the bulk modulus of the material. On the other hand, a purely deviatoric perturbation has its image on the minor axis of the stress envelope, that provides then a representation of the shear modulus. This also explains the very narrow shape of the our ellipses: as our numerical probe experiments are carried out with zero contact friction, we detect very small (but non-zero) shear moduli.
When looking to figure 3(e), the images of both isotropic and deviatoric strain increments do not lie anymore on the axes of the ellipse, but they are rather in an off-axes location. This is due to the anisotropy in the system. In fact, when a volumetric strain is applied to an anisotropic configuration, along with the volumetric stress response (bulk modulus) also a shear stress response develops. The corresponding 


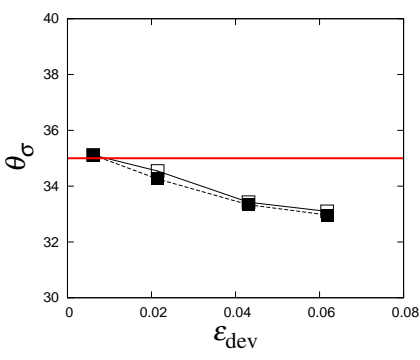

(a)

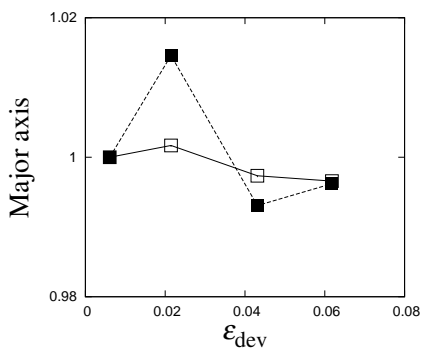

(c)

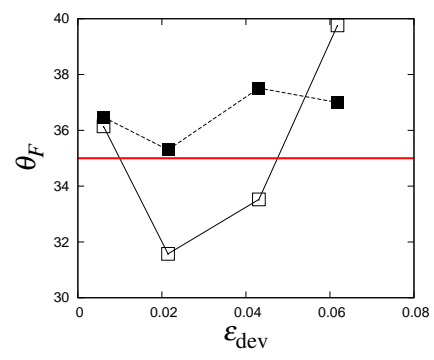

(b)

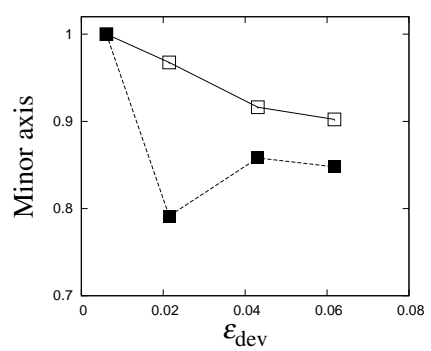

(d)
Figure 7: Evolution of inclination of the response envelope of (a) stress and (b) fabric relative to the isotropic packing response with deviatoric strain. The red horizontal line represents isotropic compression (I.C. $35^{\circ}$ ) in the strain space. Evolution of the (c) the major axis and (d) minor axis of the elliptical stress response envelope relative to the isotropic packing response with deviatoric strain (undrained axisymmetric path). Open and solid symbols represent elastic (Fig. 3) and plastic (Fig. 5) response respectively. The stress response envelopes are fitted with ellipses, while the fabric response envelopes are fitted with straight lines to extract the inclination. Points corresponding to the configurations in Figs. 3(e), 4(e), 5(e) and 6(e) are omitted.

stress vector can be decomposed in two components along the major and minor axes directions, that give a measure of the bulk modulus and the cross-coupling anisotropic stiffness, respectively (Kumar 2014). Finally we highlight that, in any isotropic/anisotropic configuration, the major and minor axis of the ellipse depict the magnitude and direction (in stress- or fabricspace), i.e the composition of the response, of maximum and minimum stiffness of the system.

We extend this type of analysis and, together with the stress response envelope, we plot the fabric response envelope to study the incremental fabric response due to a given strain increment and relate it to the stress behavior (Thornton 2010, Zhao 2013). An interesting question concerns the elastic range in granular materials and the allowed amplitude of the applied perturbation such the response remains elastic (Sibille 2009, Froiio 2010, Calvetti 2003). We use the response envelopes of stress and fabric to address this topic.

\subsection{Results: Elastic regime}

In figures 3 and 4 we report the resulting envelopes of stress and fabric for the whole set of samples at $-\varepsilon_{\mathrm{dev}}=0.006,0.021,0.043,0.068$ and 0.32 . Figs. 3(a)-3(e) show that, as long as $\|\delta \varepsilon\|<10^{-4}$, the response envelope saves the elliptical shape along the whole axisymmetric path with the ellipse centered at a the origin of the stress increment plane, meaning that we are looking at the incremental linear elastic behavior of the isotropic/anisotropic configurations. As anisotropy develops, the characteristics of the stress envelope change. Such modifications are reported in figures 7(a) and 7(c)-7(d), together with data for the elasto-plastic range, that will be discussed in the next session. The inclination of the major axis systematically decreases (Fig. 7(a)), that is the main path is inducing a rotation of the direction of maximum (elastic) stiffness. Figs. 7(c)-7(d) shows the change in magnitude of the major and minor axes of the elliptical envelope, as fitted from Fig. 3, with respect to the initial values. The major axis remains almost the same while the minor axis shrinks with increasing $-\varepsilon_{\mathrm{dev}}$. This is associated with the volume conserving deformation path, that leads to a reduction of the shear stiffness with respect to initial near isotropic state, while the bulk stiffness stays fairly constant (Kumar 2014).

When looking at the fabric response envelope in Fig. 4, we observe that the elastic regime, is associated with a linear fabric change in the isotropic and almost zero in the deviatoric direction and for any state (from initial-isotropic to anisotropic). However, as the sample becomes more anisotropic, the fabric envelope slightly tilts, see Fig. 7(b), as the direction of maximum fabric change deviates from the composition of strain, but in a direction opposite to stress (Weinhart 2013, Thornton 2010).

\subsection{Results: Elasto-plastic regime}

As further step, in figures 5 and 6, we plot the stress and fabric response envelopes related to larger strain increment amplitude, $\|\delta \varepsilon\|>10^{-3}$. In this case the magnitude of each vector in the stress response envelope represents the directional elasto-plastic stiffness of the material (rather than the elastic stiffness) for the associated loading direction and strain increment. Fig. 7 (a) shows that the major principal axis $\theta_{\sigma}$ rotates with increasing anisotropy, with angles very close to those measured in the elastic case. In Figs. 7(c) and 7(d) we report changes of the dimension the axis of the fitted elliptical response, with much bigger fluctuation with respect to the elastic data. The length of the minor axis (elasto-plastic shear stiffness) reduces - faster than the purely elastic case, while the major axis remains almost unchanged, like in the purely elastic range. In the final steady state, the data collapse on a unique line, shifted with respect to the center of the stress space, meaning that the plastic shear stiffness (minor axis) drops to zero as expected. While the inclination of the ellipse depends solely on the elastic contribution, the shift in figure 5(e) is an indication of incremental nonlinearity, as predicted for rate-type constitutive models 
(Wu 2000). Please notice that, for the sake of clarity, points corresponding to the steady state configuration are omitted in Fig. 7.

Finally, we look at the fabric response in Fig. 6 for large strain $\|\delta \varepsilon\|>10^{-3}$. While no rearrangements have been observed in figure 4 (elastic regime), we depict a finite value of fabric change in this regime, associated with plasticity (Kumar 2014). Besides the fluctuations, $\theta_{F}$ seems first to increase with increasing anisotropy and finally saturate around $35^{\circ}$ in the steady state, corresponding to the isotropic compression in the strain space. From comparison of Figs. 4(e) and 6(e) we learn that two situations are always associated with zero fabric change, the elastic regime and any steady state (Kumar 2014). Similarly to the incremental stress in figure 5, the center of the line in figure 6(e) shows a displacement with respect to the center of the space. The inclination of the elasto-plastic fabric envelope is reported in Fig. 7(b), but no clear trend about the orientation of the maximum fabric-change direction can be inferred.

\section{CONCLUSIONS}

We have studied the small strain behavior of granular materials, by building stress and fabric response envelopes for isotropic and anisotropic samples. From our analysis, we obtain indications about the change in the orientational stiffness of the material and the tilt in the direction of maximum stiffness with increasing anisotropy. We are able to distinguish between elastic and elasto-plastic regimes, where stress and structure responses are qualitatively different. The anisotropy of the sample affects the tilt of the response envelope, due to the cross-coupling of the isotropic and deviatoric terms. Our study provides a fundament for the development and validation of constitutive models involving fabric evolution, which is the next topic to be addressed. Extension of the work to more general deformation paths (including also plane-strain) and the analysis of the dependence on the initial volume fraction is in progress. Adding more realistic material behavior like friction is another step to come closer to real geomaterials, however, the present data provide the frictionless limit as reference case.

\section{ACKNOWLEDGEMENTS}

We thank Jia Lin and Wei Wu (BOKU, Vienna) for scientific discussions. This work is financially supported by the European Union funded Marie Curie Initial Training Network, FP7 (ITN-238577), and by the NWO-STW VICI grant 10828.

\section{REFERENCES}

F. Alonso-Marroquín, S. Luding, H. J. Herrmann, and I. Vardoulakis (2005). Role of anisotropy in the elastoplastic response of a polygonal packing. Phys. Rev. E., 71(5), 051304(1-18).

J.P. Bardet (1994). Numerical simulations of the incremental responses of idealized granular materials. Int. J. of Plasticity, 10(8), 879-904.

F. Calvetti, G. Viggiani, and C. Tamagnini (2003). A numerical investigation of the incremental behavior of granular soils. Rivista Italiana di Geotecnica 37(3), 11-29.

P. G. de Gennes (1999). Granular matter: a tentative view. Rev. Mod. Phys. 71(2), 374D382.

I. Einav (2012). The unification of hypo-plastic and elastoplastic theories. International Journal of Solids and Structures, 49(11), 1305-1315.

A. Ezaoui and H. Di Benedetto (2009). Experimental measurements of the global anisotropic elastic behaviour of dry Hostun sand during triaxial tests, and effect of sample preparation. Geotechnique, 59(7), 621-635.

F. Froiio and J. N. Roux (2011). Incremental response of a model granular material by stress probing with DEM simulations. In Proc. Particles 2011pp. 183-197.

J. D. Goddard (1990). Nonlinear Elasticity and PressureDependent Wave Speeds in Granular Media. Proc. R. Soc. Lond. A, 430(1878), 105-131.

G. Gudheus (1979). A Comparison of Some Constitutive Laws for Soils Under Radially Symmetric Loading and Unloading. In Proc. 3rd Conf. Numerical Methods in Geomechanics, pp. 1309-1323.

O. I. Imole, N. Kumar, V. Magnanimo, and S. Luding (2013). Hydrostatic and Shear Behavior of Frictionless Granular Assemblies Under Different Deformation Conditions. KONA, 30, 84108.

N. Kumar, S. Luding \& V. Magnanimo (2014). Macroscopic model with anisotropy based on micro-macro informations. Acta Mechanica, DOI:10.1007/s00707-014-1155-8.

L. La Ragione and V. Magnanimo (2012). Contact anisotropy and coordination number for a granular assembly: A comparison of distinct-element-method simulations and theory. Phys. Rev. E., 85(3).

S. Luding (2005). Anisotropy in cohesive, frictional granular media. J. Phys. Cond. Matt., 17(24), S2623-S2640.

V. Magnanimo, L. La Ragione, J.T Jenkins, P. Wang \& H.A. Makse (2008). Characterizing the shear and bulk moduli of an idealized granular material. Europhys. Lett., 81, 34006.

D. Muir Wood, T. Sadek, L. Dihoru, M.L. Lings, H. Javaheri (2006). Deviatoric stress response envelopes from multiaxial tests on sand. In Soil Stress Behavior: Measurements, Modeling and Analysis, Springer, pp. 253-262.

L. Sibille, F. Nicot, F.-V. Donzoe, and F. Darve (2009). Analysis of failure occurrence from direct simulations. Eur. J. Env. Civ. Eng., 13(2), 187-201.

C. Thornton and L. Zhang (2010). On the evolution of stress and microstructure during general 3D deviatoric straining of granular media. Géotechnique, 60(5), 333-341.

T. Weinhart, R. Hartkamp, A.R. Thornton and S. Luding (2013). Coarse-grained local and objective continuum description of three-dimensional granular flows down an inclined surface. Phys. Fluids, 25, 070605.

W. Wu and D. Kolymbas. Hypoplasticity then and now (2000). In Constitutive Modelling of Granular Materials, SpringerVerlag, pp. 57-101.

J. Zhao and N. Guo (2013). Unique critical state characteristics in granular media considering fabric anisotropy. Géotechnique, 63(8), 695-704. 